\title{
PPAR y Protects Against Hypoxic Renal Tubular Epithelial Cell Injury in Rat Model: A Randomized Controlled Trial
}

\section{Shi-Qun Zhu}

Guangxi Medical University

Jia-Sen Zou

Guangxi Medical University

\section{Xiu-Qi Chen}

Guangxi Medical University

Li Tu

Guangxi Medical University

Shu-Juan Jian

Guangxi Medical University

Yuan-Han Qin ( $\sim$ qinyuanhan603@163.com )

Guangxi Medical University

\section{Research Article}

Keywords: peroxisome proliferator-activated receptor $y$, hypoxia injury, kidney injury

Posted Date: March 2nd, 2022

DOI: https://doi.org/10.21203/rs.3.rs-1299965/v1

License: (1) This work is licensed under a Creative Commons Attribution 4.0 International License.

Read Full License 


\section{Abstract \\ Background}

To explore $t$ function of peroxisome proliferator-activated receptor y(PPARY) in renal tissue in acute hypoxic renal rat model injury.

\section{Methods}

24 male SD rats were randomly divided into normal control group, PPARy agonist group (rosiglitazone 10 $\mathrm{mg} / \mathrm{kg} . \mathrm{d}$ ), PPARy inhibitor group $\square \mathrm{GW} 9662,1 \mathrm{mg} / \mathrm{kg} \cdot \mathrm{d}$ (and hypoxia injury group, with six rats in each group. The normal control group without any treatment, the other three groups were exposed to $7500 \mathrm{~m}$ altitude for seven hours of acute hypobaric hypoxia. The mRNA and protein expressions of PPARy, superoxide dismutase (SOD), interleukin-1 $\beta \square \mathrm{IL}-1 \beta \mathrm{D}$ and renal endothelin(ET-1) in renal tissue were detected by using real-time fluorescence quantitative polymerase chain reaction (RT-qPCR) and western blotting. The kidney's morphology was observed by light microscope and electron microscope.

\section{Results}

The mRNA and protein expression levels of PPARy and SOD in hypoxia injury group decreased significantly $(P<0.05)$, while the mRNA and protein expression levels of IL-1 $\beta$ and ET-1 increased significantly compared with the normal control group $(P<0.05)$. After intervention with PPAR agonists, the PPARY and SOD were elevated significantly, while IL-1 $\beta$ and ET-1 were decreased significantly compared to the hypoxia injury group. The renal tubule epithelial cells (RTEC) were less damaged and abscission was reduced.

\section{Conclusions}

PPARy protect renal tubular epithelial cells from hypoxia-induced injury. PPARy agonists can be used as potential target interventions to alleviate acute hypoxic kidney injury.

\section{Introduction}

The kidney is very sensitive to hypoxia; once the hypoxia stress is irreversible, it will lead to kidney damage. Renal hypoxia is considered to play an important role in the development of various renal diseases $^{[1]}$. Acute kidney injury (AKI) or chronic kidney disease (CKD) in different diseases are induced by alterations in renal blood flow ${ }^{[2]}$.

Peroxisome proliferator-activated receptors (PPARs) are a family of nuclear transcription factors, which belong to the type $₫$ nuclear hormone receptor superfamily. According to the different encoding genes, 
PPARs can be divided into three cell subtypes, namely PPARa, PPAR $\beta$ and PPARY ${ }^{[3]}$. Each subtype has its specific agonists ${ }^{[4]}$, and there are significant differences in the expression distribution of these three subtypes. PPARy expression is distributed in kidney, adipose tissue and immune system, and is involved in normal kidney development and lipid metabolism. PPARy has physiological functions such as regulating water and salt reabsorption, regulating renal blood flow and activating renin angiotensin

system $^{[5,6]}$. In recent years, it has been found that the change of PPARy receptor expression level is involved in the occurrence and development of various kidney diseases. But the PPARY in hypoxiainduced renal injury was few reported. In this study, a rat model of acute hypoxia-induced renal injury was established, and PPARy receptor expression was interfered with by PPARy agonist rosiglitazone and inhibitorGW9662. To exploret role of PPARy in renal tissue in acute hypoxic renal rat model injury.

\section{Methods}

\section{Animal grouping and intervention}

24 rats were randomly divided into normal control group, PPARy agonist group, PPARy inhibitor group and hypoxia injury group, with six rats in each group. PPARY agonist group and PPARy inhibitor group were given rosiglitazone $10 \mathrm{mg} / \mathrm{kg} \cdot \mathrm{d}, \mathrm{GW} 9662,1 \mathrm{mg} / \mathrm{kg} \cdot \mathrm{d}$ respectively, intraperitoneal injection three days before hypoxia until the end of the experiment. Normal control group did not receive any intervention treatment, and was given food or water. The other three groups were placed in the low-pressure and hypoxic animal chamber, and the simulated altitude in the chamber was raised to $7500 \mathrm{~m}$ (oxygen concentration was about $9.0 \%$ ). The ambient temperature in the simulated cabin was $25 \pm 2{ }^{\circ} \mathrm{C}$, the humidity was measured at $55 \%$, and the subjects were given food and water in the cabin. After seven hours of hypoxia, the rats were put down. After the renal capsule and renal fascia were removed from the left kidney tissue, part of the tissue was placed in $4 \%$ paraformaldehyde for pathology, and the other part was placed at $-80^{\circ} \mathrm{C}$ for subsequent experiments.

\section{Renal Histopathological Examination By Light Microscope And Transmission Electron Microscopy (Tem)}

The kidney tissues of different parts, about the size of soybeans, were fixed with $10 \%$ neutral formalin and made into $3 \sim 4 \mu \mathrm{m}$ thick paraffin sections according to the conventional method. After staining with haematoxylin and eosin (HE), the kidney tissues were observed under ordinary light microscope. The lateral section of the removed left kidney was dissected, and the tissue with a diameter of $1 \mu \mathrm{m}$ was cut from the renal cortex. The tissue was fixed with $3.1 \%$ glutaraldehyde, bathed in buffer solution, fixed with $1 \%$ osmium acid, dehydrated by acetone and embedded with Epon812, and then prepared a semi-thin section. They were positioned under a light microscope, then cut into ultra-thin sections, dyed with uranium acetate and aluminum citrate, and observed under TEM. 


\section{Quantitative Real-time Fluorescent Pcr}

Total RNA was extracted from kidney tissue by NucleoZOL (Macherey-Nagel, Germany), the mRNA was reversely transcribed into cDNA according to the instructions of TaKaRa reverse transcription kit, and amplified by ABI 7500 PCR machine. The primers were designed and synthesized by Sangon Bioengineering (Shanghai) Co., LTD., and their sequences are shown in Table 1. Total PCR reaction system: forward and reverse primer $0.4 \mu \mathrm{L}$, Mix $10.0 \mu \mathrm{L}$, plate $2.0 \mu \mathrm{L}$, adding ddHO to $20.0 \mu \mathrm{L}$. Reaction conditions: $95^{\circ} \mathrm{C}$, 30s, one cycle; $95^{\circ} \mathrm{C}, 10 \mathrm{~s}, 60{ }^{\circ} \mathrm{C}, 30 \mathrm{~s}, 40$ cycles; $95^{\circ} \mathrm{C}, 15 \mathrm{~s}, 60^{\circ} \mathrm{C}, 60 \mathrm{~s}, 95^{\circ} \mathrm{C}, 15 \mathrm{~s}$, cycle once. The relative mRNA expression was normalized to $\beta$-actin and calculated using the $2^{-} \Delta \triangle \mathrm{Ct}$ formula.

Table 1

The real-time PCR primer of rat mRNA

\begin{tabular}{|lll|}
\hline Gene & Forward primer $\left(\mathbf{5}^{\prime} \rightarrow \mathbf{3}^{\prime}\right)$ & Reverse primer $\left(\mathbf{5}^{\prime} \rightarrow \mathbf{3}^{\prime}\right)$ \\
\hline PPARY & CCATCGAGGACATCCAAGACAACC & GTGCTCTGTGACAATCTGCCTGAG \\
\hline IL-1 $\beta$ & CTCACAGCAGCATCTCGACAAGAG & TCCACGGGCAAGACATAGGTAGC \\
\hline ET-1 & TGCTCCTGCTCCTCCTTGATGG & TCGCTTAGACTTAGAAGGGCTTCC \\
SOD & CCACATCGGCCTGTGTATATCCCAG & CGTGAAGCTGGAGAAGGAGAAGCTG \\
\hline B-actin & AGTGTGACGTTGACATCCGTA & GCCAGAGCAGTAATCTCCTFCT \\
\hline
\end{tabular}

\section{Western blot analysis}

Total protein was extracted from kidney tissue with protein lysate. After boiling and denaturation, $10 \mu \mathrm{g}$ of protein was added to sample, and protein was isolated by sodium dodecyl sulfate polyacrylamide gel (SDS-PAGE). Protein was transferred to polyvinylidene fluoride (PVDF) membrane by wet transfer method. The membranes were blocked with $1 \times$ TBST containing $5 \%$ nonfat milk for $1.5 \mathrm{~h}$ at room temperature. Subsequently, the membrane was added with primary antibody PPARY $(1: 1,000)$, IL-1 $\beta$ $(1: 1,000)$, ET-1 $(1: 1,500)$ and SOD $(1: 2,000)$ and incubated overnight in a refrigerator at $4{ }^{\circ} \mathrm{C}$. Washed the membrane with TBST three times for 10 minutes each. The membranes were added with horseradish peroxidase labeled secondary antibody (1:5 000, SAB Company) and incubated at room temperature for $1.5 \mathrm{~h}$ and washed three times for $10 \mathrm{~min}$ each. The membrane was scanned using fluO-RChem ${ }^{\circledR} \mathrm{HD} 2$ imaging system and the results were analyzed using ImageJ software. The ratio of gray value of target protein band to gray value of internal reference $\beta$-actin protein band was used as the relative expression level of target protein.

\section{Statistical analysis}

Statistical analyses were carried out using the software package SPSS version 25. All experimental data are shown as the Mean \pm The standard deviation. The significant differences between two groups were evaluated using the independent-samples test. Moreover, the significant differences among groups were 
examined using one-way analysis of variance (ANOVA) with post-hoc Fisher's LSD, $P<0.05$ was considered to indicate a statistically significance.

\section{Results}

\section{Pathological changes of renal tissue}

Hematoxylin-eosin staining showed that there was no significant change in renal histopathology in normal control group. Compared with the normal control group, the RTEC were swollen and exfoliated in both PPARy inhibitor group and hypoxia injury group, but the PPARy inhibitor group showed more severe cell damage. The swelling degree of RTEC was reduced in PPARy agonist group compared with hypoxia injury group. TEM showed mitochondrial swelling and stromal focal edema of renal tubular epithelial cells and glomerular podocytes in both PPARy inhibitor group and hypoxia injury group compared with the normal control group. The mitochondrial swelling and stromal focal edema was remission in the PPARy agonist group (Figure 1).

\section{The Mrna Expression Levels Of Ppary, Sod, II-1 $\beta$ And Et-1 By Rt-pcr}

To investigate the effects of rosiglitazone, a PPARy agonist, on acute hypoxic renal injury in rats, we detected the expression levels of PPARY, SOD, IL-1 $\beta$ and ET-1. Compared with normal control group, the mRNA expressions of PPARY and SOD in hypoxia injury group were significantly decreased (Fig. 2, $\mathrm{P}<$ 0.05 ), while the mRNA expressions of IL-1 $\beta$ and ET-1 were significantly increased (Fig. $2, P<0.05$ ). Compared with hypoxia injury group, the mRNA expression levels of PPARY and SOD were significantly increased (Fig. 2, P < 0.05), while the mRNA expression levels of IL-1 $\beta$ and ET-1 were significantly decreased (Fig. 2, P < 0.05) in the PPARy agonist group. In the PPARy inhibitor group, the mRNA and protein expressions of PPARY and SOD were more decreased, and IL-1 $\beta$ and ET- 1 were more increased compare to the hypoxia injury group (Fig. $2, \mathrm{P}<0.05$ ).

\section{The Protein Expression Levels Of Ppary, Sod, II-1 $\beta$ And Et-1 By Wester-blot}

The wester-blot result showed that protein expression levels of PPARY, SOD, IL-1 $\beta$ and ET-1. Compared with normal control group, the protein expressions of PPARY and SOD in hypoxia injury group were significantly decreased (Fig. 3, P< 0.05), while the IL-1 $\beta$ and ET-1 were significantly increased (Fig. 3, P< 0.05). Compared with hypoxia injury group, the PPARY and SOD were significantly increased (Fig. $3, \mathrm{P}<$ $0.05)$, while IL-1 $\beta$ and ET-1 were significantly decreased $(P<0.05)$ in the PPARY agonist group. In the PPARY inhibitor group, the PPARY and SOD were more decreased, and IL-1 $\beta$ and ET-1 were more increased compare to the hypoxia injury group (Fig. $3, \mathrm{P}<0.05)$. 


\section{Discussion}

Studies have shown that hypoxia is one of the most common causes of renal tubular epithelial cell injury, so oxidative injury plays an important role in the occurrence and development of renal diseases ${ }^{[7,8]}$. This present study showed that low oxygen induced renal injury in rats with acute hypoxia model, using low pressure hypoxic animals lab to make oxygen, establish acute kidney injury model of rats induced by hypoxia. HE staining showed that the epithelial cells of renal tubules were swollen and exfoliated in the model group. And TEM showed that RTEC and glomerular podocyte mitochondria were significantly swollen after hypoxia compared with normal control group, suggesting that the model was successfully constructed. The result was similar to the Chhabra ${ }^{[9]}$ study, in which the renal tissue structural damage was observed in the acute hypoxia group for one day. Mitochondria are the main organelles eukaryotic cells powered, renal tubular epithelial cells in high-energy mitochondrial content is very rich. The causes of renal hypoxia include systemic hypoxia caused by hypoxic environment and local oxygen deficiency of kidney caused by different diseases such as atherosclerosis and hypertension. Current research models mainly focus on local renal hypoxia, which changes renal blood flow. The mechanism of systemic hypoxia in renal injury remains unclear. This study was conducted with the model of systemic hypoxia in rats.

In the present study, expressions of PPARy was decreased and RTEC was injure in hypoxia injury group and PPARY inhibitor group. But the PPARY agonist relieved the injury degree of RTEC and glomerular podoccytes, suggesting that the change of PPARy expression level may be related to the injury of RTEC.Activated PPAR- $y$ can inhibit adhesion molecules, chemokines and other inflammatory factors, inhibit inflammatory response, reduce reactive oxygen species and oxidative stress ${ }^{[10,11]}$. Mice with PPARy gene knockout developed renal insufficiency, reduced creatinine clearance, fibrosis, and tubular dilation ${ }^{[12]}$. It was reported that pioglitazone, a PPARy agonist, alleviated renal tissue injury by upregulating the expression of PPARy and increasing antioxidant capacity in rats with ischemia-reperfusion injury ${ }^{[13-15]}$. In diabetic mice, the activation of amino-terminal kinase (JNK) pathway up-regulates the expression of PPARY and inhibits the apoptosis of islet cells and mesangial cells, thereby improving insulin resistance and delaying renal injury ${ }^{[16]}$. In the RTEC model induced by sodium urate crystals, the expression of intracellular inflammatory bodies and interleukin-1 $\beta$ decreased significantly after PPARY activation, and the cell damage was alleviated ${ }^{[17]}$. In hypoxia-induced RTEC injury, the agonist rosiglitazone can up-regulate the protein expression of PPARy and reduce RTEC injury. PPARy expression in damaged RTEC was inhibited in the hypoxia/reoxygenation (HR) model.

In this study, we established a rat model of acute hypoxic kidney injury and used PPARy agonist rosiglitazone to intervene the expression of PPARy. The results showed that after PPARY agonist intervention, the expressions of PPARY and SOD were significantly increased, while the expressions of IL$1 \beta$ and ET-1 were significantly decreased, suggesting that up-regulation of PPARY expression can alleviate hypoxia-induced renal injury in rats. SOD, IL-1 $\beta$ and ET-1 were involved in the process of hypoxic kidney injury. SOD is the most important antioxidant enzyme in the body, which is involved in maintaining 
cell integrity and metabolic process. The level of SOD consumption in kidney tissue reflects the degree of oxidative damage in kidney tissue. Studies have shown that in cisplatin-induced liver injury model, SOD

and PPARy expression in liver were decreased ${ }^{[18]}$. The result was similar in our acute hypoxic kidney injury rat. Acute hypoxia rapidly activates endothelial cells and triggers an inflammatory response ${ }^{[19]}$. IL$1 \beta$ is the most common proinflammatory factor and plays an important role in inflammatory response. If the renal tubule injury, endothelial cell function can be impaired by the production of inflammatory mediators (such as IL-1 $\beta$ ). PPAR- $\gamma$ agonists can inhibit IL-1 $\beta$ induced expression of vascular smooth muscle cells to secrete hemagglutinin-like receptor $1^{[20]}$. ET-1 is secreted by vascular endothelial cells. Hypoxia can increase ET synthesis. Renal vessels are known to be 10 times more sensitive to the vasoconstriction effect of ET than other vascular beds. It has been reported that the secretion of ET-1 is increased in PPARY -knockout mice ${ }^{[21]}$. It is suggested that PPARy may be directly involved in inhibiting the secretion of ET-1. Our study suggested that the decline of PPARY was involved in the development of hypoxic kidney injury, PPARY agonists maybe protect against hypoxic kidney injury by inhibiting the ET-1 and IL-1ß.

\section{Conclusions}

PPARy expression was highly correlated with acute hypoxia kidney injury in rat model. PPARy agonist can alleviate hypoxic kidney injury in a possible pathway that inhibited the ET-1 and IL-1 $\beta$ expression.

\section{Abbreviations}

AKI

Acute kidney injury

ANOVA

Analysis of variance

CKD

Chronic kidney disease

ET

Endothelin

HE

Hematoxylin and eosin

IL

interleukin

PPAR

peroxisome proliferator-activated receptor

PVDF

polyvinylidene fluoride

RTEC

renal tubule epithelial cells 
SD

Sprague-Dawley

SDS-PAGE

sodium dodecyl sulfate polyacrylamide gel

SOD

superoxide dismutase

TBST

TBS (tris buffered saline) + Tween

TEM

transmission electron microscopy

\section{Declarations}

\section{Ethics approval and consent to participate[}

The study was carried out in compliance with the ARRIVE guidelines 2.0. The animal usage and protocol were reviewed and approved by Guangxi Medical University Ethical Review Committee. Animal Use License No. : SYXK GUI 2020-0004; Animal production License No. : SCXK GUI 2020-0003.And the experimental operations and procedures were in accordance with the regulations of the People's Republic of China on the administration of experimental animals and the ethical requirements.

\section{Consent for publication $\square$}

Not applicable.

\section{Availability of data and materials $\square$}

The datasets used and/or analyzed during the current study are available from the corresponding author on reasonable request. Our data is deposited in 10.6084/m9.figshare.19233777.

\section{Competing interests $\square$}

The authors declare that they have no competing interests.

\section{Funding:}

This work was supported by the Guangxi Medical High-level Backbone Talent "139" Plan under Grant [No.G201901010].

\section{Author's contributions:}

SQZ and JSZ conceived the study and contributed equally to the paper, SQZ, JSZ, LT and SJJ collected and analyzed the data, SQZ wrote the manuscript. XQC revised the final paper. YHQ contributed to the conception and design of the study. All authors read and approved the final manuscript. 
Acknowledgements:

Not applicable.

\section{References}

1. Evans RG. Introduction: Renal Hypoxia in Kidney Disease. Seminars in nephrology 2019; 39(6):517519.

2. Legrand M, Mik EG, Johannes T, Payen $D$, Ince $C$. Renal hypoxia and dysoxia after reperfusion of the ischemic kidney. Molecular medicine (Cambridge, Mass) 2008; 14(7-8):502-516.

3. Han L, Shen WJ, Bittner S, Kraemer FB, Azhar S. PPARs: regulators of metabolism and as therapeutic targets in cardiovascular disease. Part II: PPAR- $\beta / \delta$ and PPAR- $y$. Future cardiology $2017 ; 13(3): 279-$ 296.

4. Poulsen L, Siersbæk M, Mandrup S. PPARs: fatty acid sensors controlling metabolism. Seminars in cell \& developmental biology 2012; 23(6):631-639.

5. Corrales P, Izquierdo-Lahuerta A, Medina-Gómez G. Maintenance of Kidney Metabolic Homeostasis by PPAR Gamma. International journal of molecular sciences 2018; 19(7).

6. Medina-Gomez G, Gray SL, Yetukuri L, Shimomura K, Virtue S, Campbell M, et al. PPAR gamma 2 prevents lipotoxicity by controlling adipose tissue expandability and peripheral lipid metabolism. PLoS genetics 2007; 3(4):e64.

7. Shu S, Wang Y, Zheng M, Liu Z, Cai J, Tang C, et al. Hypoxia and Hypoxia-Inducible Factors in Kidney Injury and Repair. Cells 2019; 8(3).

8. de Seigneux S, Martin PY. Preventing the Progression of AKI to CKD: The Role of Mitochondria. Journal of the American Society of Nephrology: JASN 2017; 28(5):1327-1329.

9. Chhabra V, Anand AS, Baidya AK, Malik SM, Kohli E, Reddy MPK. Hypobaric hypoxia induced renal damage is mediated by altering redox pathway. PloS one 2018; 13(7):e0195701.

10. Villapol S. Roles of Peroxisome Proliferator-Activated Receptor Gamma on Brain and Peripheral Inflammation. Cellular and molecular neurobiology 2018; 38(1):121-132.

11. Ahmadian M, Suh JM, Hah N, Liddle C, Atkins AR, Downes M, et al. PPARY signaling and metabolism: the good, the bad and the future. Nature medicine 2013; 19(5):557-566.

12. Toffoli B, Gilardi F, Winkler C, Soderberg M, Kowalczuk L, Arsenijevic Y, et al. Nephropathy in Ppargnull mice highlights PPARY systemic activities in metabolism and in the immune system. PloS one 2017; 12(2):e0171474.

13. Elshazly S, Soliman E. PPAR gamma agonist, pioglitazone, rescues liver damage induced by renal ischemia/reperfusion injury. Toxicology and applied pharmacology 2019; 362:86-94.

14. Reel B, Guzeloglu M, Bagriyanik A, Atmaca S, Aykut K, Albayrak G, et al. The effects of PPAR-Y agonist pioglitazone on renal ischemia/reperfusion injury in rats. The Journal of surgical research 2013; 182(1):176-184. 
15. Ragab D, Abdallah DM, El-Abhar HS. Cilostazol renoprotective effect: modulation of PPAR-y, NGAL, KIM-1 and IL-18 underlies its novel effect in a model of ischemia-reperfusion. PloS one 2014; 9(5):e95313.

16. Koh ES, Lim JH, Kim MY, Chung S, Shin SJ, Choi BS, et al. Anthocyanin-rich Seoritae extract ameliorates renal lipotoxicity via activation of AMP-activated protein kinase in diabetic mice. Journal of translational medicine 2015; 13:203.

17. Hong W, Hu S, Zou J, Xiao J, Zhang X, Fu C, et al. Peroxisome proliferator-activated receptor Y prevents the production of NOD-like receptor family, pyrin domain containing 3 inflammasome and interleukin $1 \beta$ in HK-2 renal tubular epithelial cells stimulated by monosodium urate crystals. Molecular medicine reports 2015; 12(4):6221-6226.

18. Mansour HH, El Kiki SM, Galal SM. Metformin and low dose radiation modulates cisplatin-induced oxidative injury in rat via PPAR-y and MAPK pathways. Archives of biochemistry and biophysics 2017; 616:13-19.

19. Gonzalez NC, Wood JG. Alveolar hypoxia-induced systemic inflammation: what low PO(2) does and does not do. Advances in experimental medicine and biology 2010; 662:27-32.

20. Hofnagel O, Luechtenborg B, Stolle K, Lorkowski S, Eschert H, Plenz G, et al. Proinflammatory cytokines regulate LOX-1 expression in vascular smooth muscle cells. Arteriosclerosis, thrombosis, and vascular biology 2004; 24(10):1789-1795.

21. Kang BY, Park KK, Kleinhenz JM, Murphy TC, Green DE, Bijli KM, et al. Peroxisome ProliferatorActivated Receptor $y$ and microRNA 98 in Hypoxia-Induced Endothelin-1 Signaling. American journal of respiratory cell and molecular biology 2016; 54(1):136-146.

\section{Figures}



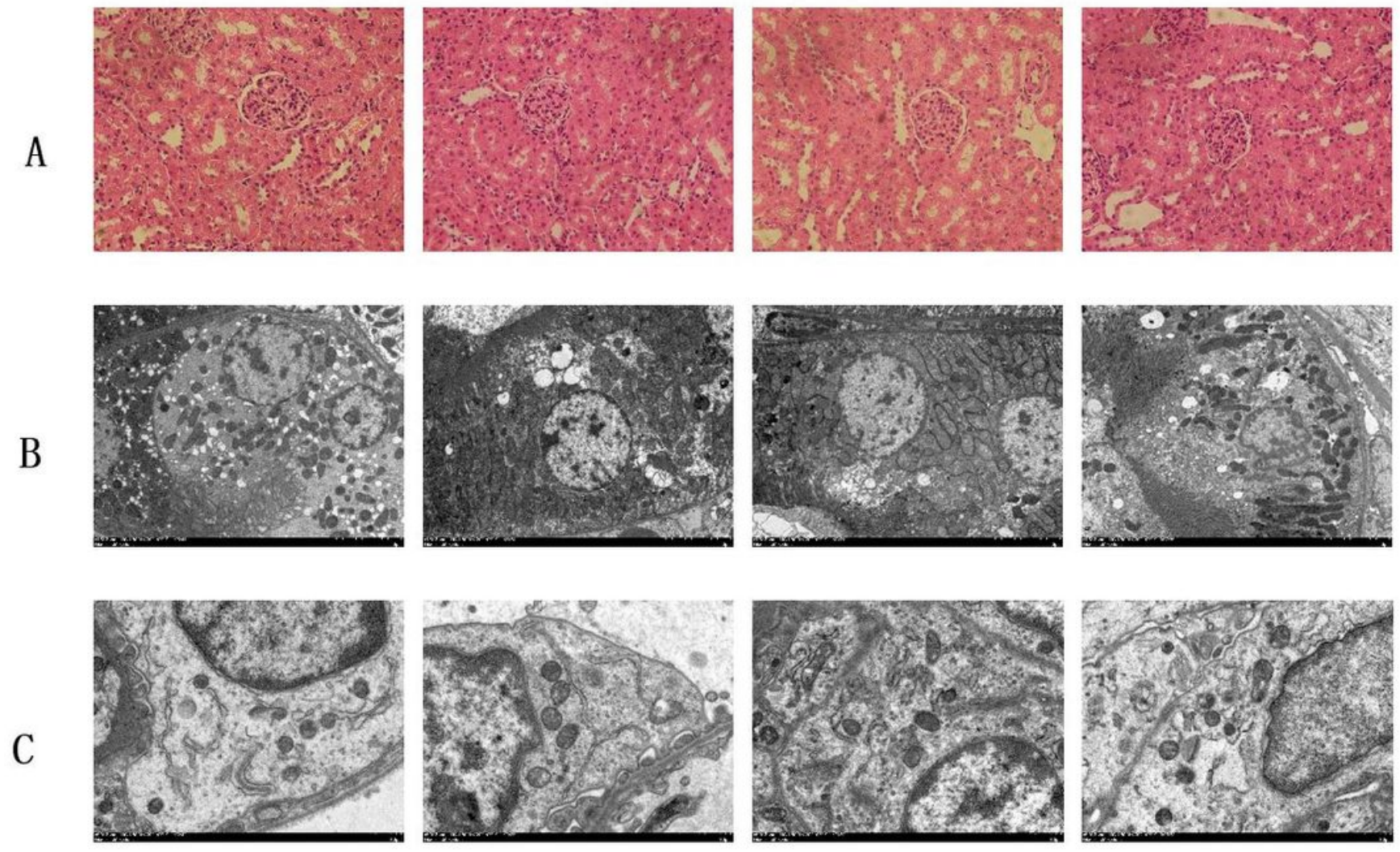

$\mathrm{N}$

$\mathrm{H}$

$\mathrm{R}$

G

\section{Figure 1}

Pathological changes of renal tissue. (A) Pathological changes of rat kidney with HE staining under light microscope $(\times 400)$. (B-C) Under the transmission electron microscopy of rat renal tubular epithelial cells $(\times 1.5 \mathrm{k})$, and rat glomerular podocytes ( $\times 6 \mathrm{k})$. N: normal control group; $\mathrm{H}$ : hypoxia injury group; R: PPARY agonist group;G: PPARY inhibitor group. 


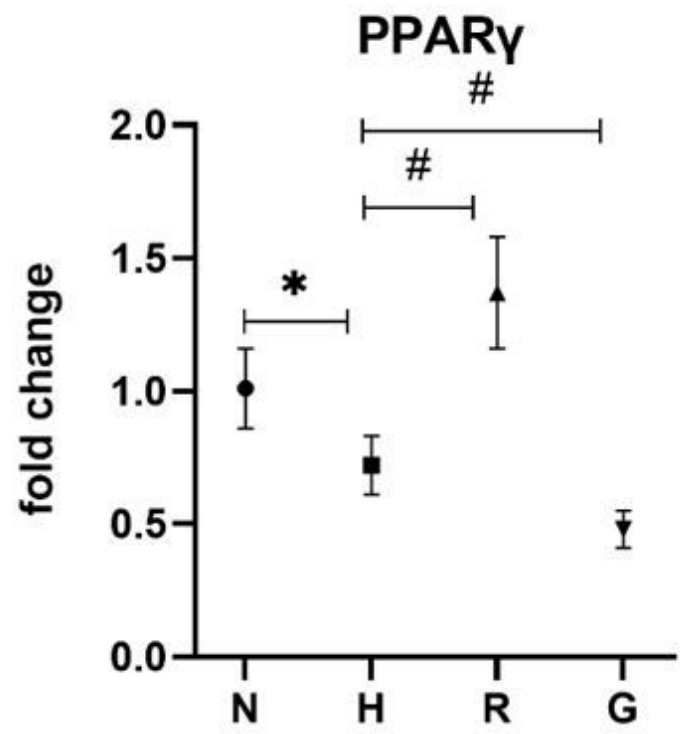

SOD
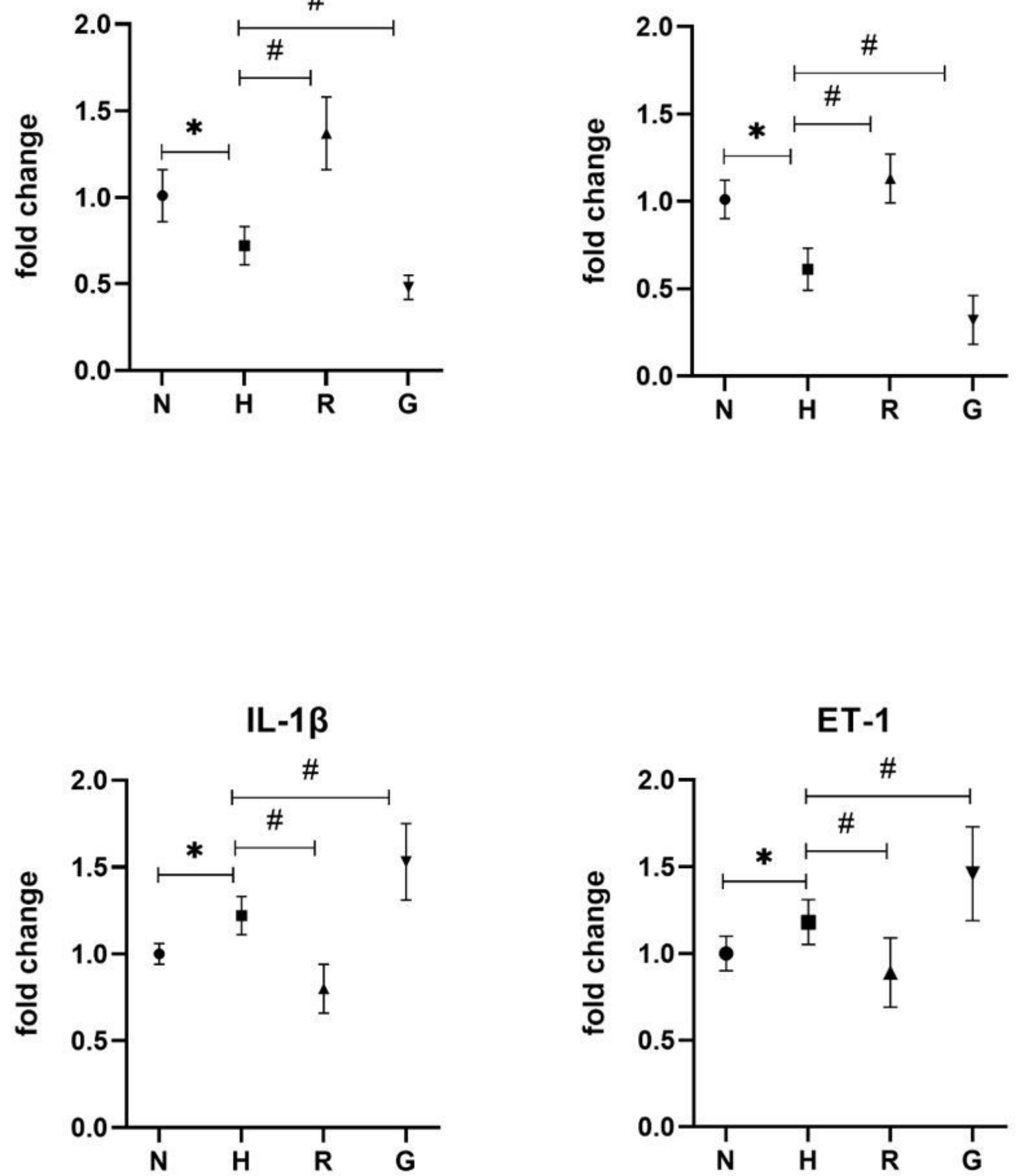

Figure 2

The mRNA expression levels of PPARY区SODヌIL-1 $\beta$ and ET-1. RT-PCR analysis of expressions of PPARY囚 SOD $\mathbb{\text { IL-1 }} \beta$ and ET-1 in rat kidney tissue. ${ }^{*} \mathrm{P}<0.05$, compared with normal control group; \# $\mathrm{P}<0.05$, compared with hypoxia injury group. $\mathrm{N}$ : normal control group; $\mathrm{H}$ : hypoxia injury group; R: PPARy agonist group;G: PPARy inhibitor group. 

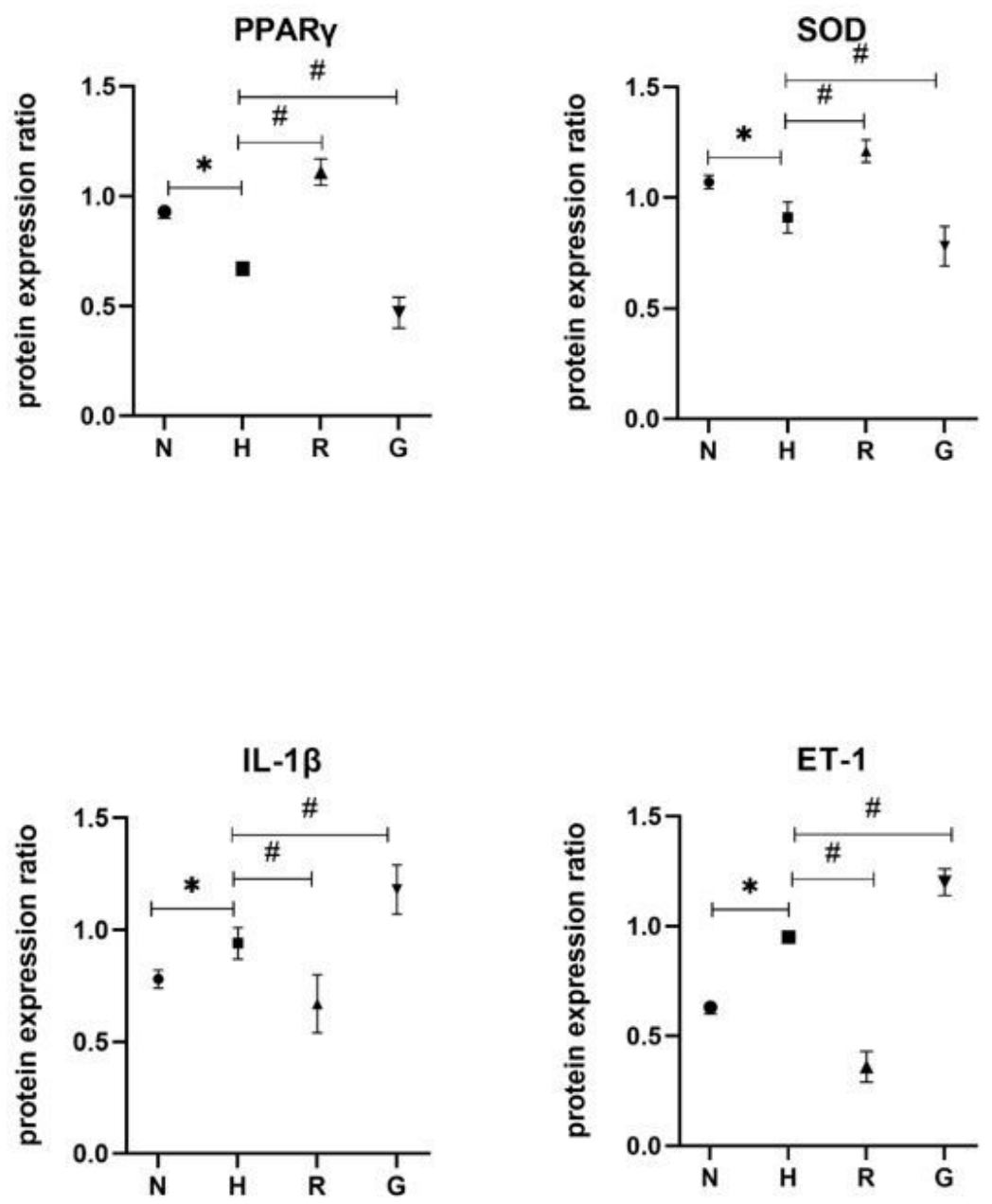

Figure 3-1
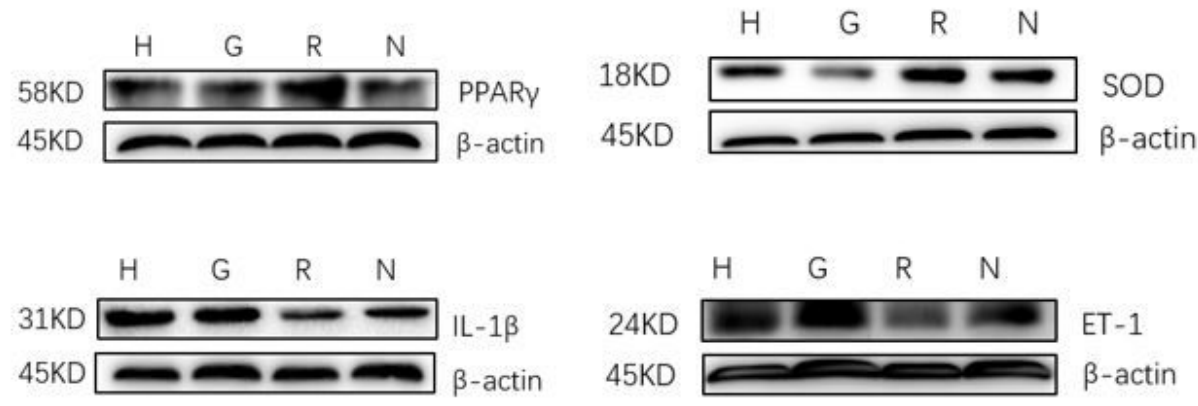

Figure 3-2

\section{Figure 3}

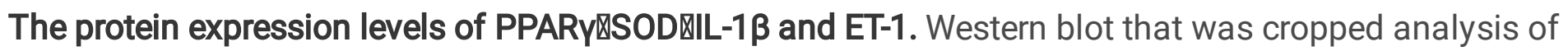
expressions of PPARY $\triangle S O D \otimes I L-1 \beta$ and ET- 1 in rat kidney tissue. ${ }^{*} P<0.05$, compared with normal control group; \# $\mathrm{P}<0.05$, compared with hypoxia injury group. $\mathrm{N}$ : normal control group; $\mathrm{H}$ : hypoxia injury group; R: PPARy agonist group;G: PPARy inhibitor group. The blots were cropped, full-length blots are presented in Supplementary Figure 1. 


\section{Supplementary Files}

This is a list of supplementary files associated with this preprint. Click to download.

- SupplementaryFigure1.docx 